\title{
177Lu-Labeled Near-infrared Upconversion Nanoparticles for targeted theranostics of Breast Cancer Lymphatic Metastases
}

\section{Chuan Zhang}

Soochow University

\section{Yujuan Zhang}

Soochow University

\section{Maolin Liang}

Soochow University

\section{Xiumin Shi}

Soochow University

\section{Longfei Fan}

Soochow University

\section{Kai Yang}

Soochow University

\section{Feng Wang}

Nanjing First Hospital

Wei Li

Second Affiliated Hospital of Soochow University

Ran Zhu ( $\nabla$ zhuran@suda.edu.cn )

Soochow University https://orcid.org/0000-0001-9351-4702

\section{Research Article}

Keywords: Breast Cancer, Lymph node metastasis, Radiotherapy, Upconversion nanoparticles

Posted Date: February 1st, 2022

DOI: https://doi.org/10.21203/rs.3.rs-1268077/v1

License: (c) (i) This work is licensed under a Creative Commons Attribution 4.0 International License. Read Full License 


\section{Abstract}

The theranostics of lymph node metastasis has always been one of the major obstacles to defeating breast cancer and an important decisive factor in the prognosis of patients. Herein, we design $\mathrm{NaGdF}_{4}: \mathrm{Yb}, \mathrm{Tm} @ N a L u F 4$ upconversion nanoparticles with PEG and anti-HER2 monoclonal antibody (trastuzumab, Herceptin) (NP-mAb) conjugation for targeting lymphatic metastasis. Radionuclides ${ }^{177} \mathrm{Lu}$ could be chelated by the bisphosphate groups of NP-mAb. The obtained ${ }^{177}$ Lu-NP-mAb exhibited excellent radiolabeling stability and show high accumulation and prolong retention in the lymph node metastasis after intratumoral injection into the footpad by SPECT/CT imaging. Utilizing the $\beta$ rays released by ${ }^{177} \mathrm{Lu},{ }^{177} \mathrm{Lu}-\mathrm{NP}-\mathrm{mAb}$ could not only decrease the number of lymph node metastasis, but also significantly improve therapeutic efficiency of lymph node metastasis. Additionally, ${ }^{177}$ Lu-NP-mAb induce no obvious toxicity to treated mice through blood routine, live and spleen function assay. Therefore, ${ }^{177}$ Lu-NP-mAb we designed could be acted as excellent theranostics agents for lymph node metastasis, providing a potential alternative treatment option for lymph node metastasis therapy.

\section{Introduction}

The metastasis is an extremely severe step in the progression of tumors. Approximately $90 \%$ of deaths in cancer patients are due to metastasis rather than the primary tumor ${ }^{[1]}$. The lymphatic system is the preferred route for metastasis of most solid tumors in vivo, and draining lymph nodes are usually the earliest sites and the first station of metastasis ${ }^{[2,3]}$. Lymph node metastasis often indicates a poor prognosis, and is an important indicator of cancer progression ${ }^{[4,5]}$. The dissection and local radiotherapy of sentinel lymph nodes can significantly improve the prognosis of patients with lymph node metastasis, further emphasizing the importance of the theranostics of lymph node metastasis ${ }^{[6-8]}$. Recently, breast cancer has replaced lung cancer as the most common cancer worldwide ${ }^{[9]}$. Similar to other solid tumors, lymph node metastasis plays a major role in promoting the invasion and metastasis of breast cancer. Therefore, reducing the occurrence of lymph node metastasis and removing tumor cells from the lymphatic system are the keys for combating breast cancer.

The current treatment methods for lymph node metastasis mainly include surgical resection, local radiotherapy and chemotherapy. Surgical resection as an invasive treatment have been widely used to perform regional or sentinel lymph node dissection. However, the efficiency of surgical resection usually depends on the diagnostic techniques of lymph nodes. Clinically, methylene blue dye, technetium colloid or fluorescent dye ICG are commonly used to locate and detect sentinel lymph nodes for guiding lymph node resection ${ }^{[10-13]}$. However, these methods have certain shortcomings and limitations. It is difficult to detect the deep lymph nodes and distinguish the lymph node metastasis or normal lymph nodes. According to the literature, local radiotherapy had been used for advanced patients with metastases to supraclavicular lymph nodes, reducing the risk of local recurrence and improving the overall survival ${ }^{[14}$, 15]. However, the inevitable risks of exposure to radiation of the heart, lungs, and skin can lead to events such as acute radiation skin damage, lung damage, and increased incidence of heart disease ${ }^{[16-19]}$. 
Similar to radiotherapy, chemotherapy is usually applied in the advanced patients with breast cancer. Recently, the neoadjuvant chemotherapy have been developed to reduce the size of the primary tumor and eliminate minor peripheral lesions before the surgery or radiotherapy, further improving the patient's prognosis and quality of life ${ }^{[20]}$. Chemotherapeutic agents based on small molecular often result in poor lymphatic absorption in clinical applications, affecting the long-retention in lymph node metastasis [21, ${ }^{22]}$. More importantly, the systemic side effects of chemotherapy are very obvious ${ }^{[23]}$. Therefore, it is an urgent need to develop new personalized theranostics treatment of lymph node metastasis in breast cancer.

Given its suitable size and surface properties, nanomaterials can freely enter the intercellular matrix ${ }^{\text {[24] }}$. During the competitive absorption process between lymphatics and blood vessels, some nanomaterials will preferentially enter the lymphatic vessels from the interstitium [25-27]. Therefore, interstitially administration (subcutaneous, intratumoral or peritumoral) of nanodrugs exhibit great potential to target the lymphatic system. The delivery of nanodrugs through the lymphatic system effectively targets and accumulates in lymph node metastasis, avoiding the rapid drug clearance caused by direct ingestion by the blood system and further reducing the risk of toxicity. Utilizing the nanomaterials as nano-drug carriers could optimize drug accumulation in solid tumor sites, achieving high efficient and selective lymphatic system enrichment by subcutaneous administration ${ }^{[28-30]}$. Although number of papers have reported that nano-drug combined with small molecule drugs using the principle of lymphatic transport could kill the cancer cells aggregated in the lymph node site, the combination of nano-drug with radioisotope therapy for lymphatic system has not been reported.

The passive targeting of nano-drugs based on the enhanced penetration and retention (EPR) effect is strongly influenced by tumor heterogeneity. For the metastatic tumor, nano-drugs without tumor targeting ability could induce the non-specific distribution and unnecessary side effects ${ }^{[31,32]}$. An effective way to overcome these passive targeting limitations is to introduce targeting ligands or antibodies on the surface of nanoparticles for improving the cancer cells uptake of nanodrugs through their active binding ability to receptors or antigens specifically expressed on the tumor cell surface ${ }^{[33-35]}$. Human epidermal growth factor receptor 2 (HER2)-positive breast cancer is an aggressive type of breast cancer that tends to grow more rapidly and spread more easily. Anti-HER2 therapies such as Herceptin (trastuzumab), are highly effective in the clinic, significantly improving the prognosis of patients with HER2-positive breast cancer ${ }^{[36,37]}$. In addition, trastuzumab has been widely used in the development of various nanodrugs for the diagnosis and treatment of HER2-positive breast cancer ${ }^{[38,39]}$. Therefore, in this study, we synthesized rare-earth upconversion nanoparticle conjugated with trastuzumab (NP-mAb). The obtained NP-mAb could be efficiently labeled with therapeutic radioisotope ${ }^{177} \mathrm{Lu}$ ( ${ }^{177} \mathrm{Lu}-\mathrm{NP}-\mathrm{mAb}$ ) (half-life of $6.71 \mathrm{~d}$ ) through simple chelation, which could be used for radioisotope therapy of HER2- positive breast cancer lymph node metastasis tumor in mice. ${ }^{177}$ Lu-NP-mAb was intratumorally injected into the footpad area, and then moved into the lymph node metastasis via the delivery of lymphatic system, effectively inhibiting the occurrence and growth of tumour in the footpad area and lymph node metastasis, further prolonging the survival of treated mice (Figure 1). Importantly, such therapeutic strategy our developed 
even exhibited no obvious side effects on the blood system, liver and kidney function of mice. Therefore, our developed strategy will provide a new method for treatment of lymphatic metastases.

\section{Results And Discussions}

In this work, we developed radionuclides ${ }^{177}$ Lu labeled trastuzumab antibody conjugated UCNP (NaGdF4:Yb,Tm@NaLuF4) nanoparticles for lymph nodes metastatic tumor treatment (Figure 2a). The

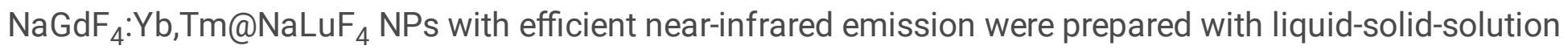
(LSS) solvothermal synthesis method ${ }^{[40-43]}$. Transmission electron microscopy (TEM) imaging showed that UCNP nanoparticles were monodisperse spherical with an average size of $22.03 \pm 1.82 \mathrm{~nm}$ (Figure $2 \mathrm{~b}$, Figure S1). To improve its biocompatibility, UCNP nanoparticles were modified with polyethylene glycol with a maleimide group. The dynamic light scattering (DLS) assay showed that the PEGylated UCNP nanoparticles were measured to be $50 \mathrm{~nm}$ and exhibited a narrow hydrodynamic size distribution in water, suggesting that the mal-PEG-DP ligands could effectively replace the oleic acid ligands without inducing nanoparticles aggregation (Figure $2 \mathrm{c}$ ). In order to effectively target breast cancer lymph node metastasis, anti-HER2 monoclonal antibody was conjugated to UCNP NPs (NP-mAb) via a reaction between maleimide group on the PEG ligand and sulfhydryl residues of the partially reduced anti-HER2 monoclonal antibody. The size of NP-mAb was measured to be $60 \mathrm{~nm}$ through DLS assay (Figure 2c). Moreover, the zeta potential of the UCNP nanoparticles before and after mAb conjugation was changed from $5.68 \mathrm{mV}$ to $12.63 \mathrm{mV}$ (Figure S2). The above results strongly demonstrated that the monoclonal antibody molecules were successfully coupled on the surface of the UCNP nanoparticles. Under the excitation of 980-nm laser, the NP-mAb mainly emitted near infrared at 804 nm (Figure 2d).

Besides, we also investigated the stability of NP-mAb nanoparticles in the different solutions. The results showed that NP-mAb nanoparticles exhibited excellent colloidal stability in pure water and 10\% FBS, and no nanoparticles aggregation was observed for more than 3 days (Figure 2e-f; Figure S3-4). Afterwards, utilizing its bisphosphate groups, the NP-mAb was labeled with radionuclides ${ }^{177}$ Lu by chelation. The radiolabeling yield of ${ }^{177}$ Lu- NP-mAb was about $>95 \%$ through thin-layer paper chromatography assay (Figure $2 \mathrm{~g}$ ). The radiolabeling stability of ${ }^{177}$ Lu-NP-mAb also exhibited great well in PBS solution (Figure S5).

We next studied the cytotoxicity of UCNP nanoparticles to HER2 positive human breast carcinoma cell line (SKBR3) cells and human liver cell line (HL7702) cells by the CCK-8 assay. As shown in Figure 3a, after incubating with 5 mM NP-mAb for $24 \mathrm{~h}$, the viabilities of SKBR3 cells and HL7702 cells were higher than $80 \%$, ensuring the bio-safety of NP-mAb. In order to verify the targeting capability of the NP-mAb nanoparticles, the cellular uptake was conducted. Human breast cancer cell line SKBR3 cells with high HER2 expression were incubated with NPs, NP-mAb and ${ }^{177}$ Lu-NP-mAb for $12 \mathrm{~h}$, respectively, and the HER2 low-expressed triple-negative human breast carcinoma cell line MDA-MB 231 cells were also incubated with ${ }^{177} \mathrm{Lu}-\mathrm{NP}-\mathrm{mAb}$ for $12 \mathrm{~h}$. Under $980 \mathrm{~nm}$ laser irradiation, the upconversion fluorescence signal of cells in different treated group was observed. The results showed that the upconversion 
fluorescence of SKBR3 + NP-mAb group was significantly stronger than other groups (Figure 3b). Although the labeling of ${ }^{177}$ Lu resulted in decreased uptake of NP-mAb in SKBR3 cells, it was still higher than that of SKBR3+ NPs and MDA-MB $231+$ NP-mAb group (Figure S6). In addition, optical imaging (IVIS Spectrum imaging system) also showed that the uptake of the nanoparticles in SKBR3 + NP-mAb group was significantly higher than that in SKBR3+ NPs group. Meanwhile, free anti-HER2 monoclonal antibody was firstly added to cell culture medium and significantly inhibited the uptake of NP-mAb by SKBR3 cells (Figure S7). These results further demonstrated that NP-mAb could target SKBR3 cells through targeting the HER2 receptor.

After radionuclides labelling, the cytotoxicity effect of ${ }^{177}$ Lu-NP-mAb was also investigated. SKBR3 cells were incubated with different concentration of free ${ }^{177}$ Lu, ${ }^{177}$ Lu-NPs and ${ }^{177}$ Lu-NP-mAb for $24 \mathrm{~h}$, respectively. The cell viabilities were measured by CCK-8 assay. The results showed that the inhibitory effect of ${ }^{177}$ Lu-NP-mAb on SKBR3 cells was significantly higher than that of free ${ }^{177}$ Lu and ${ }^{177}$ Lu-NPs (Figure 3c). Meanwhile, the DNA damage of SKBR3 cells treated with ${ }^{177} \mathrm{Lu}-\mathrm{NP}-\mathrm{mAb}$ nanoparticles was also studied. The $\mathrm{Y}-\mathrm{H} 2 \mathrm{AX}$ fluorescence imaging showed that ${ }^{177} \mathrm{Lu}-\mathrm{NP}-\mathrm{mAb}$ induced significantly higher DNA damage than other groups (Figure 3d, Figure S8).

Next, we evaluated the in vivo imaging of lymphatic metastasis using ${ }^{177}$ Lu-NP-mAb nanoparticles as nanoprobes. The HER2-positive breast cancer lymphatic metastasis model was established according to the literatures ${ }^{[44,45]}$. Mice bearing SKBR3 lymphatic metastasis were intratumorally injected with ${ }^{177}$ LuNP-mAb and ${ }^{177}$ Lu-NP in the footpad, respectively, and imaged by a small animal SPECT/CT imaging system. As shown in Figure 4a, ${ }^{177}$ Lu-NP-mAb treated mice exhibited significant accumulation in the lymph node metastasis at $12 \mathrm{~h}$ post injection, compared with ${ }^{177} \mathrm{Lu}-\mathrm{NP}$ treated mice. The quantitative analysis of the radioactive intensity in the injection site and lymph node metastasis by PMOD 3.6 software (PMOD Technologies, Zurich, Switzerland) suggested that the accumulation of ${ }^{177}$ Lu-NP-mAb at the metastatic lymph nodes site was approximately $10 \% \mathrm{ID} / \mathrm{g}$, which was significantly higher than that of ${ }^{177}$ Lu-NPs (Figure 4b). After 7 days of observation, the retention time of ${ }^{177}$ Lu-NP-mAb in lymph node metastasis was significantly prolonged compared with ${ }^{177}$ Lu-NPs. The ratio of radioactive intensity of ${ }^{177}$ Lu-NPs and ${ }^{177}$ Lu-NP-mAb in lymph node metastasis and primary tumors showed that the UCNP conjugated with anti-HER2 monoclonal antibody exhibited high accumulation in lymph node metastasis, further indicating that ${ }^{177}$ Lu-NP-mAb might be an excellent drug candidate for the treatment of lymph node metastasis (Figure 4c).

Based on the excellent accumulation and retention of ${ }^{177}$ Lu-NP-mAb in lymph node metastasis, we monitored and evaluated the therapeutic effect of ${ }^{177}$ Lu-NP-mAb on lymph node metastasis. The timeline of construction of tumor model, drug administration and outcome analysis was shown in Figure 5a. After 2 weeks of inoculation of the tumor cells into SPF-grade nude mice, mice were imaged by micro-MRI to determine the formation of lymph node metastasis. As showed in figure $5 \mathrm{~b}$, we could clearly observe the primary tumor and lymphatic metastasis through MR imaging. Afterwards, the primary tumor in the 
footpad was treated with different nanoparticles, including PBS, NP-mAb, free ${ }^{177}$ Lu, ${ }^{177}$ Lu-NPs and ${ }^{177}$ Lu-NP-mAb. The number of lymph node metastases was reduced in both ${ }^{177}$ Lu-NP-mAb and ${ }^{177}$ LuNPs-treated groups compared with the PBS group (Figure $5 \mathrm{c}$ ). Owing to the higher uptake and longer retention of NP-mAb in lymph node metastasis, ${ }^{177}$ Lu-NP-mAb exhibited significant inhibition effect for lymph node metastasis. Additionally, the rate of lymph node metastasis was slightly decreased in the free ${ }^{177}$ Lu treated group, which might be due to the antitumor effect of $\beta$-rays emitted by ${ }^{177}$ Lu. In contrast, there was no change in the metastatic rate of lymph nodes in the NP-mAb treated group. We also collected the primary tumor and lymph node metastasis to record the volume of tumor. As shown in Figure 5d, the primary tumor volumes of mice treated with ${ }^{177}$ Lu-NP-mAb was smaller than that of the other four groups, which might be related to the better distribution and intracellular uptake of ${ }^{177} \mathrm{Lu}-\mathrm{NP}$ $\mathrm{mAb}$ within the tumor. From the lymph node metastasis volume analysis, both ${ }^{177}$ Lu-NPs and ${ }^{177}$ Lu-NP$\mathrm{mAb}$ could inhibit the tumor growth, suggesting that the nanoparticles could enter into the lymphatic system and accumulate in the lymph node. Moreover, the inhibitory effect of ${ }^{177}$ Lu-NP-mAb group was better than that of the ${ }^{177}$ Lu-NPs group, demonstrating that anti-HER2 antibody could improve the accumulation and retention of nanoparticles in lymph nodes.

Besides, we also investigated the potential mechanism of radioisotope therapy in lymph node metastasis. Lymph nodes from mice with different treatments were collected for HE staining, TUNEL staining and CD44 immunohistochemistry (IHC) after 2 weeks post injection. HE staining and TUNEL staining of lymph nodes exhibited that ${ }^{177}$ Lu-NP-mAb induced most severe apoptosis compared with other control groups (Figure 6a). CD44 is widely expressed on the surface of breast tumor stem cells ${ }^{[46]}$. CD44 IHC results of lymph node metastasis showed that CD44 expression level in the ${ }^{177}$ Lu-NP-mAb treated group was significantly decreased compared with the PBS group, indicating that the tumor stem cells in lymph node metastasis decreased after ${ }^{177}$ Lu-NP-mAb treatment. The flow cytometry of tumor cells collected from mice with different treatments also indicated the reduced CD44 expression in the ${ }^{177}$ Lu-NP-mAb treated group (Figure 6b). In addition, epithelial cell adhesion molecule (EpCAM) expressed on the surface of most epithelial tumor cells, including breast cancer cells, is an important marker in circulating tumor cell detection (CTC). The flow cytometry results showed that the expression level of EpCAM in the ${ }^{177}$ Lu-NP-mAb treated group was significantly lower than that in PBS group (Figure 6c). Therefore, we designed ${ }^{177}$ Lu-NP-mAb could significantly inhibited the lymph node metastasis and reduce the incidence of tumor metastasis.

The potential side-effect of ${ }^{177}$ Lu-NP-mAb was also investigated. The blood samples collected from mice with different treatments were used to evaluate the routine blood tests, liver and kidney function. As showed in Figure S9-11, there was no significant change in blood indexes such as white blood cell count (WBC), red blood cell (RBC), platelet count (PLT) and hemoglobin (HGB) in ${ }^{177}$ Lu, ${ }^{177}$ Lu-NPs and ${ }^{177}$ LuNP-mAb groups. In addition, the results of liver function (alanine aminotransferase, ALT; aspartate aminotransferase, AST; alkaline phosphatase, ALP; gamma glutamyl transpeptidase, GGT) and renal function (blood urea, UREA; creatinine, CREA) were no significant difference between the groups, 
indicating that ${ }^{177} \mathrm{Lu},{ }^{177} \mathrm{Lu}-\mathrm{NPs}$ and ${ }^{177} \mathrm{Lu}-\mathrm{NP}-\mathrm{mAb}$ had no obvious liver and kidney toxicity. Therefore, ${ }^{177}$ Lu-NP-mAb we designed could act as an excellent therapeutic agent for lymph node metastasis without side-effects.

\section{Conclusion}

In this work, we successfully developed ${ }^{177}$ Lu labeled trastuzumab conjugated upconversion nanoparticles for lymph node metastasis theranostics. SPECT/CT imaging showed that ${ }^{177}$ Lu-NP-mAb exhibited high accumulation and prolonged retention in lymph node metastasis. Importantly, the injected ${ }^{177}$ Lu-NP-mAb significantly reduced the occurrence of lymph node metastasis and inhibited the growth of lymph node metastasis. In addition, ${ }^{177}$ Lu-NP-mAb induced no obvious side-effect to treated mice though the blood routine, liver and spleen function assay. Therefore, this strategy we developed could not only decrease the number of lymph node metastasis, but also significantly improve therapeutic efficiency of lymph node metastasis compared with the traditional treatment, offering a potential alternative treatment option for lymph node metastasis therapy.

\section{Materials And Methods}

\section{Preparation of ${ }^{177}$ Lu-NP-mAb}

The detailed preparation of $\mathrm{NaGdF}_{4}: \mathrm{Yb}, \mathrm{Tm}_{\mathrm{N}} \mathrm{NaLuF}_{4}$ nanoparticles was provided in the Supporting Information. NP-mAb conjugation was prepared according to the previously reported methods ${ }^{[47]}{ }^{177} \mathrm{LuCl}_{3}-\mathrm{HCl}$ solution $(20 \mu \mathrm{L}, 18.5-37 \mathrm{MBq})$ dissolved in $20 \mu \mathrm{L}$ of $0.25 \mathrm{M}$ sodium acetate ( $\mathrm{NaOAc}$ ) was added into the NP-mAb solution $(1 \mathrm{mg} / \mathrm{mL}, 0.05 \mathrm{~mL})$ and then stirred at room temperature for $30 \mathrm{~min}$. The mixture was centrifuged at $3000 \mathrm{r} / \mathrm{min}$ for $5 \mathrm{~min}$ three times through $100 \mathrm{~K}$ ultrafiltration tube to remove the excess ${ }^{177} \mathrm{Lu}$. The radiochemical purity of ${ }^{177} \mathrm{Lu}-\mathrm{NP}-\mathrm{mAb}$ was measured by paper chromatography (mobile phase: EDTA).

\section{In vitro experiments}

The human breast cancer cell line SKBR3, MDA-MB-231 and human normal liver cell line HL-7702 were obtained from Cell Source Center, Chinese Academy of Science (Shanghai, China). SKBR3 cells were cultured and passaged in Dulbecco's Modified Eagle Medium (DMEM) supplemented with $10 \%$ fetal bovine serum (FBS), penicillin $(100 \mathrm{U} / \mathrm{mL})$, and streptomycin $(0.1 \mathrm{mg} / \mathrm{mL})$. MDA-MB-231 cells and HL7702 cells were cultured and passaged in Roswell Park Memorial Institute 1640 Medium (RPMI-1640) supplemented with $10 \%$ fetal bovine serum (FBS), penicillin $(100 \mathrm{U} / \mathrm{mL})$, and streptomycin $(0.1 \mathrm{mg} / \mathrm{mL})$.

For in vitro potential toxicity assay, SKBR3 cells and HL-7702 cells were seeded in 96-well plates $\left(5 \times 10^{3}\right.$ cells/well) and cultured at $37^{\circ} \mathrm{C}$ overnight, respectively. The different concentrations of NP-mAb ( 0 , $0.04,0.08,0.16,0.31,0.63,1.25,2.5$ and $5 \mathrm{mM}$ ) were added and cultured for $24 \mathrm{~h}$. The cell culture medium 
was replaced by fresh medium in each well, and the cells were incubated for another 24 or $48 \mathrm{~h}$. Cell viability was measured by the Cell Counting Kit-8 (CCK-8) assay.

For in vitro radioisotope therapy, SKBR3 cells were seeded in 96 -well plates $\left(5 \times 10^{3}\right.$ cells $/$ well $)$ and cultured at $37^{\circ} \mathrm{C}$ overnight. The different concentrations of free ${ }^{177} \mathrm{Lu},{ }^{177} \mathrm{Lu}-\mathrm{NPs}$ and ${ }^{177} \mathrm{Lu}-\mathrm{NP}-\mathrm{mAb}(0$, $0.3,0.6,1.2,2.5,5,10,20$ and $40 \mathrm{mCi}$ ) were added and then incubated for $24 \mathrm{~h}$. The cell culture medium was replaced by fresh medium in each well, and the cells were incubated for another 24 or $48 \mathrm{~h}$. Cell viability was measured by the Cell Counting Kit-8 (CCK-8) assay.

For cell uptake experiments, SKBR3 cells and MDA-MB-231 cells were seeded onto a glass-bottom cell culture dish ( $\$ 15 \mathrm{~mm}, \mathrm{NEST})$ at densities of $5 \times 10^{4}$ cells/well, respectively. After $12 \mathrm{~h}$, SKBR3 cells were treated with NP-mAb, ${ }^{177}$ Lu- NP-mAb and NPs, respectively. MDA-MB-231 cells were treated with NP-mAb. After another $12 \mathrm{~h}$ of incubation, cells were stained with 4',6-diamidino-2-phenylindole (DAPI) and imaged by confocal microscopy (FV1200, OLYMPUS, Tokyo, Japan).

For $\mathrm{y}-\mathrm{H} 2 \mathrm{AX}$ staining studies, SKBR3 cells were seeded onto a glass-bottom cell culture dish ( $\$ 15 \mathrm{~mm}$, NEST) at densities of $5 \times 10^{4}$ cells/well. Cells were treated with PBS (control), NP-mAb, free ${ }^{177} \mathrm{Lu},{ }^{177} \mathrm{Lu}-$ NPs and ${ }^{177}$ Lu-NP-mAb, respectively. After $12 \mathrm{~h}$ of incubation, cells were stained with $\mathrm{y}-\mathrm{H} 2 \mathrm{AX}$ and DAPI and then imaged by confocal microscopy (FV1200, OLYMPUS, Tokyo, Japan).

\section{In vivo experiments}

Female BALB/c nude mice (5-8 weeks) were purchased from Changzhou Cavens Company. All animal experiments were performed according to the experimental animal protocols of Soochow University, and the experiments were approved by the animal ethics committee of Soochow University.

To create the HER2-positive breast cancer lymphatic metastasis model, a single-cell suspension of $2 \times$ $10^{6}$ SKBR3 cells in $20 \mu \mathrm{L}$ PBS was injected into the left footpad area of BALB/c nude mice. The popliteal lymph nodes could be palpated for enlargement and hardening after about 2 weeks, demonstrating that SKBR3 cells were successfully metastasized to the popliteal lymph nodes.

For in vivo SPECT imaging and bio-distribution studies, mice bearing SKBR3 tumor were injected in the footpad with ${ }^{177} \mathrm{Lu}-\mathrm{NP}-\mathrm{mAb}$ or ${ }^{177} \mathrm{Lu}$ - NPs at radioactive dose of $50 \mu \mathrm{Ci}$. A small animal SPECT/CT scanner (U-SPECT/CT, MILabs, Netherlands) was performed at different time points injection ( $1 \mathrm{~h}, 2 \mathrm{~h}, 5 \mathrm{~h}$, $8 \mathrm{~h}, 12 \mathrm{~h}, 24 \mathrm{~h}, 2 \mathrm{D}, 3 \mathrm{D}, 5 \mathrm{D}$ and 7D). The imaging data were reconstructed using the two-dimensional ordered subsets-expectation maximization algorithm. Volume rendered images were generated using professional PMOD software (MILabs).

Mice bearing SKBR3 lymphatic metastasis model were raised two weeks and then assigned into five groups including 1) PBS (control group), 2) NP-mAb treated group, 3) free ${ }^{177}$ Lu treated group (3.7 MBq per mouse), 4) ${ }^{177} \mathrm{Lu}$ - NPs treated group (3.7 MBq per mouse) and 5) ${ }^{177} \mathrm{Lu}-\mathrm{NP}-\mathrm{mAb}$ treated group (3.7 MBq per mouse corresponding to 50 ug of NP-mAb). These samples were injected in the left footpad area 
$(n=8)$, while the control group were administrated with $0.02 \mathrm{~mL}$ of PBS $(n=8)$. After another two weeks, micro-MRI was used to measure the tumor volume in the foot pads and lymph node metastasis. The tumor volumes were calculated using the formula: ()$^{[48,49]}$. When the tumor volume reached $2 \mathrm{~cm}^{3}$ or the weight loss exceeded $20 \%$, the experiment was terminated and the animals were sacrificed.

\section{Safety Assessment}

For immunofluorescence staining, the lymph node metastasis obtained from mice with different treatments including PBS, NP-mAb, free ${ }^{177} \mathrm{Lu},{ }^{177} \mathrm{Lu}-\mathrm{NPs}$ and ${ }^{177} \mathrm{Lu}-\mathrm{NP}-\mathrm{mAb}$ were sliced and stained with anti-CD44 (abcam, Clone: EPR18668). Hematoxylin \& eosin (H\&E) staining and terminal deoxynucleotidyl transferase dUTP nick end labelling (TUNEL) assay also were carried out to evaluate morphologic changes and apoptosis of SKBR3 tumor cells.

The blood routine, liver function and kidney function were investigated to evaluate the side effects of mice with different treatment. Blood routine assay including WBC, RBC, PLT and HGB were measured by XN-1000 F Automated Hematology Analyzer (KOBE, JAPAN). Liver and kidney functions including ALT, AST, ALP, GGT, UREA and CREA were measured by Clinical Chemistry Analyzer BS-420®Mindray, China囚.

To study the antigen expression of SKBR3 cancer cells in vivo, immunocytochemistry was used to detect cell surface antigens. The lymph node metastasis obtained from mice with different treatments including PBS, NP-mAb, free ${ }^{177} \mathrm{Lu},{ }^{177} \mathrm{Lu}-\mathrm{NPs}$ and ${ }^{177} \mathrm{Lu}-\mathrm{NP}-\mathrm{mAb}$ were homogenized in PBS (pH 7.4) containing $1 \%$ Fetal Bovine Serum (FBS) to acquire cell suspension. Afterwards, the SKBR3 cells were stained by anti-EpCAM (abcam, Clone: EPR20532-225) or anti-CD44 (abcam, Clone: EPR18668) antibodies for flow cytometry assay.

\section{Statistical analysis}

All results are expressed as means \pm SEM or SD as indicated. Multiple t-tests were used when two groups were compared.

\section{Declarations}

\section{Ethics approval and consent to participate}

Not applicable.

\section{Consent for publication}

Not applicable.

\section{Availability of data and materials}

All data related to the manuscript are available in the manuscript and supporting information. 


\section{Competing interests}

The authors declare that they have no competing interests.

\section{Funding}

This work was supported by the National Key Research Program of China (2018YFA0208800), National Natural Science Foundation of China (22076132, 21976128), the Natural Science Foundation of Jiangsu Province (BK20200100, BK20190830), Suzhou Administration of Science \& Technology (SYS2020082), Key Talents Program for medical Application of nuclear Technology (XKTJ-HRC20210012) and the Project of State Key Laboratory of Radiation Medicine and Protection, Soochow University (GZK1201806).

\section{Authors' contributions}

The manuscript was written through contributions of all authors. C.Z, Y.J.Z, and M.L.L contributed equally. All authors read and approved the final manuscript.

\section{Acknowledgements}

We also would like to thank the members in the Zhu Lab at Soochow University and the members in the Wang Lab in Nanjing First Hospital, Nanjing Medical University.

\section{References}

1. Steeg PS. Tumor metastasis: mechanistic insights and clinical challenges. Nat Med. 2006;12(8):895-904.

2. Leong SPL, Tseng WW. Micrometastatic cancer cells in lymph nodes, bone marrow, and blood: Clinical significance and biologic implications. CA Cancer J Clin. 2014;64(3):195-206.

3. Xie Y, Bagby TR, Cohen MS, Forrest ML. Drug delivery to the lymphatic system: importance in future cancer diagnosis and therapies. Expert Opin Drug Deliv. 2009;6(8):785-92.

4. Detmar M, Hirakawa S. The formation of lymphatic vessels and its importance in the setting of malignancy. J Exp Med. 2002;196(6):713-8.

5. Nathanson SD. Insights into the mechanisms of lymph node metastasis. Cancer. 2003;98(2):41323.

6. Peters J, Foord S, Dieguez C, Salvador J, Hall R, Scanlon MF. Lack of effect of the TRH related dipeptide histidyl-proline diketopiperazine on TSH and PRL secretion in normal subjects, in patients with microprolactinomas and in primary hypothyroidism. Clin Endocrinol (Oxf). 1985;23(3):289-93.

7. Giuliano AE, Hunt KK, Ballman KV, Beitsch PD, Whitworth PW, Blumencranz PW, et al. Axillary Dissection vs No Axillary Dissection in Women With Invasive Breast Cancer and Sentinel Node Metastasis: A Randomized Clinical Trial. JAMA. 2011;305(6):569-75. 
8. Donker M, van Tienhoven G, Straver ME, Meijnen P, van de Velde CJ, Mansel RE, et al. Radiotherapy or surgery of the axilla after a positive sentinel node in breast cancer (EORTC 10981-22023 AMAROS): a randomised, multicentre, open-label, phase 3 non-inferiority trial. Lancet Oncol. 2014;15(12):1303-10.

9. World Health Organization (WHO). International Agency for Research on Cancer 2020. The Global Cancer Observatory: Breast fact sheet. December, 2020. https://gco.iarc.fr/today/data/factsheets/cancers/20-Breast-fact-sheet.pdf.

10. Thongvitokomarn S, Polchai N. Indocyanine Green Fluorescence Versus Blue Dye or Radioisotope Regarding Detection Rate of Sentinel Lymph Node Biopsy and Nodes Removed in Breast Cancer: A Systematic Review and Meta-Analysis. Asian Pac J Cancer Prev. 2020;21(5):1187-95.

11. Ang CH, Tan MY, Teo C, Seah DW, Chen JC, Chan MYP, et al. Blue dye is sufficient for sentinel lymph node biopsy in breast cancer. Br J Surg. 2014;101(4):383-9.

12. Guo JJ, Yang HP, Wang S, Cao YM, Liu M, Xie F, et al. Comparison of sentinel lymph node biopsy guided by indocyanine green, blue dye, and their combination in breast cancer patients: a prospective cohort study. World J Surg Oncol. 2017;15:1477-7819.

13. Zhou Y, Li Y, Mao F, Zhang J, Zhu Q, Shen S, et al. Preliminary study of contrast-enhanced ultrasound in combination with blue dye vs. indocyanine green fluorescence, in combination with blue dye for sentinel lymph node biopsy in breast cancer. BMC Cancer. 2019;19(1):939.

14. Speers C, Pierce LJ. Postoperative Radiotherapy After Breast-Conserving Surgery for Early-Stage Breast Cancer: A Review. JAMA Oncol. 2016;2(8):1075-82.

15. Krug D, Baumann R, Budach W, Dunst J, Feyer P, Fietkau R, et al. Current controversies in radiotherapy for breast cancer. Radiat Oncol. 2017;12(1):25.

16. Darby SC, Ewertz M, McGale P, Bennet AM, Blom-Goldman U, Bronnum D, et al. Risk of Ischemic Heart Disease in Women after Radiotherapy for Breast Cancer. New Engl J Med. 2013;368(11):987-98.

17. Lettmaier S, Kreppner S, Lotter M, Walser M, Ott OJ, Fietkau R, et al. Radiation exposure of the heart, lung and skin by radiation therapy for breast cancer: A dosimetric comparison between partial breast irradiation using multicatheter brachytherapy and whole breast teletherapy. Radiother Oncol. 2011;100(2):189-94.

18. Werner EM, Eggert MC, Bohnet S, Rades D. Prevalence and Characteristics of Pneumonitis Following Irradiation of Breast Cancer. Anticancer Res. 2019;39(1791-7530):6355-8.

19. Taylor C, Correa C, Duane FK, Aznar MC, Anderson SJ, Bergh J, et al. Estimating the Risks of Breast Cancer Radiotherapy: Evidence From Modern Radiation Doses to the Lungs and Heart and From Previous Randomized Trials. J Clin Oncol. 2017;35(1527-7755):1641-9.

20. Mougalian SS, Soulos PR, Killelea BK, Lannin DR, Abu-Khalaf MM, DiGiovanna MP, et al. Use of neoadjuvant chemotherapy for patients with stage I to III breast cancer in the United States. Cancer. 2015;121(1097-0142):2544-52.

21. Ryan GM, Kaminskas LM, Bulitta JB, Mclntosh MP, Owen DJ, Porter CJH. PEGylated polylysine dendrimers increase lymphatic exposure to doxorubicin when compared to PEGylated liposomal and 
solution formulations of doxorubicin. J Controlled Release. 2013;172(1):128-36.

22. Kaminskas LM, Ascher DB, McLeod VM, Herold MJ, Le CP, Sloan EK, et al. PEGylation of interferon alpha2 improves lymphatic exposure after subcutaneous and intravenous administration and improves antitumour efficacy against lymphatic breast cancer metastases. J Controlled Release. 2013;168(2):200-8.

23. Love RR, Leventhal $H$, Easterling DV, Nerenz DR. Side effects and emotional distress during cancer chemotherapy. Cancer. 1989;63(3):604-12.

24. Hawley AE, Davis SS, Illum L. Targeting of colloids to lymph nodes: influence of lymphatic physiology and colloidal characteristics. Adv Drug Del Rev. 1995;17(1):129-48.

25. Swartz MA. The physiology of the lymphatic system. Adv Drug Del Rev. 2001;50(1):3-20.

26. Kaminskas LM, Kota J, McLeod VM, Kelly BD, Karellas P, Porter CJH. PEGylation of polylysine dendrimers improves absorption and lymphatic targeting following SC administration in rats. $\mathrm{J}$ Controlled Release. 2009;140(2):108-16.

27. Oussoren C, Zuidema J, Crommelin DJA, Storm G. Lymphatic uptake and biodistribution of liposomes after subcutaneous injection.: II. Influence of liposomal size, lipid composition and lipid dose. Biochimica et Biophysica Acta (BBA) - Biomembranes. 1997;1328(2):261-72.

28. Brannon-Peppas L, Blanchette JO. Nanoparticle and targeted systems for cancer therapy. Adv Drug Del Rev. 2012;64:206-12.

29. Alexis F, Pridgen EM, Langer R, Farokhzad OC. Nanoparticle Technologies for Cancer Therapy. Berlin Heidelberg: In: Springer; 2010. pp. 55-86.

30. Li B, Yuan Z, He Y, Hung HC, Jiang S. Zwitterionic Nanoconjugate Enables Safe and Efficient Lymphatic Drug Delivery. Nano Lett. 2020;20(6):4693-9.

31. Fang J, Nakamura H, Maeda $H$. The EPR effect: Unique features of tumor blood vessels for drug delivery, factors involved, and limitations and augmentation of the effect. Adv Drug Del Rev. 2011;63(3):136-51.

32. Maeda H. Macromolecular therapeutics in cancer treatment: The EPR effect and beyond. J Controlled Release. 2012;164(2):138-44.

33. Swain S, Sahu PK, Beg S, Babu SM. Nanoparticles for Cancer Targeting: Current and Future Directions. Curr Drug Deliv. 2016;13(8):1290-302.

34. Wang M, Thanou M. Targeting nanoparticles to cancer. Pharmacol Res. 2010;62(2):90-9.

35. Bazak R, Houri M, El Achy S, Kamel S, Refaat T. Cancer active targeting by nanoparticles: a comprehensive review of literature. J Cancer Res Clin Oncol. 2015;141(5):769-84.

36. Asif HM, Sultana S, Ahmed S, Akhtar N, Tariq M. HER-2 Positive Breast Cancer - a Mini-Review. Asian Pac J Cancer Prev. 2016;17(4):1609-15.

37. Harbeck N, Gnant M. Breast cancer. Lancet. 2017;389(10074):1134-50.

38. Chattopadhyay N, Cai Z, Pignol J-P, Keller B, Lechtman E, Bendayan R, et al. Design and Characterization of HER-2-Targeted Gold Nanoparticles for Enhanced X-radiation Treatment of 
Locally Advanced Breast Cancer. Mol Pharm. 2010;7(6):2194-206.

39. Su C-Y, Chen M, Chen L-C, Ho Y-S, Ho H-O, Lin S-Y, et al. Bispecific antibodies (anti-mPEG/anti-HER2) for active tumor targeting of docetaxel (DTX)-loaded mPEGylated nanocarriers to enhance the chemotherapeutic efficacy of HER2-overexpressing tumors. Drug Deliv. 2018;25(1):1066-79.

40. Ren F, Ding L, Liu H, Huang Q, Zhang H, Zhang L, et al. Ultra-small nanocluster mediated synthesis of $\mathrm{Nd} 3+-$ doped core-shell nanocrystals with emission in the second near-infrared window for multimodal imaging of tumor vasculature. Biomaterials. 2018;175:30-43.

41. Ren J, Jia G, Guo Y, Wang A, Xu S. Unraveling Morphology and Phase Control of NaLnF4 Upconverting Nanocrystals. J Phys Chem C. 2016;120(2):1342-51.

42. Wang $X$, Zhuang J, Peng Q, Li Y. A general strategy for nanocrystal synthesis. Nature. 2005;437(7055):121-4.

43. Johnson NJ, Korinek A, Dong C, van Veggel FC. Self-focusing by Ostwald ripening: a strategy for layer-by-layer epitaxial growth on upconverting nanocrystals. J Am Chem Soc. 2012;134(27):1106871.

44. Li X, Dong Q, Yan Z, Lu W, Feng L, Xie C, et al. MPEG-DSPE polymeric micelle for translymphatic chemotherapy of lymph node metastasis. Int J Pharm. 2015;487(1-2):8-16.

45. Long J, Luo G, Liu C, Cui X, Satoh K, Xiao Z, et al. Development of a unique mouse model for pancreatic cancer lymphatic metastasis. Int J Oncol. 2012;41(5):1662-8.

46. Al-Othman N, Alhendi A, Ihbaisha M, Barahmeh M, Alqaraleh M, Al-Momany BZ. Role of CD44 in breast cancer. Breast Dis. 2020;39(1):1-13.

47. Qiu S, Zeng J, Hou Y, Chen L, Ge J, Wen L, et al. Detection of lymph node metastasis with nearinfrared upconversion luminescent nanoprobes. Nanoscale. 2018;10(46):21772-81.

48. Dempsey MF, Condon BR, Hadley DM. Measurement of tumor "size" in recurrent malignant glioma: 1D, 2D, or 3. AJNR Am J Neuroradiol. 2005;26(4):770-6. D?.

49. Hamoud Al-Tamimi MS, Sulong G, Shuaib IL. Alpha shape theory for 3D visualization and volumetric measurement of brain tumor progression using magnetic resonance images. Magn Reson Imaging. 2015;33(6):787-803.

\section{Figures}



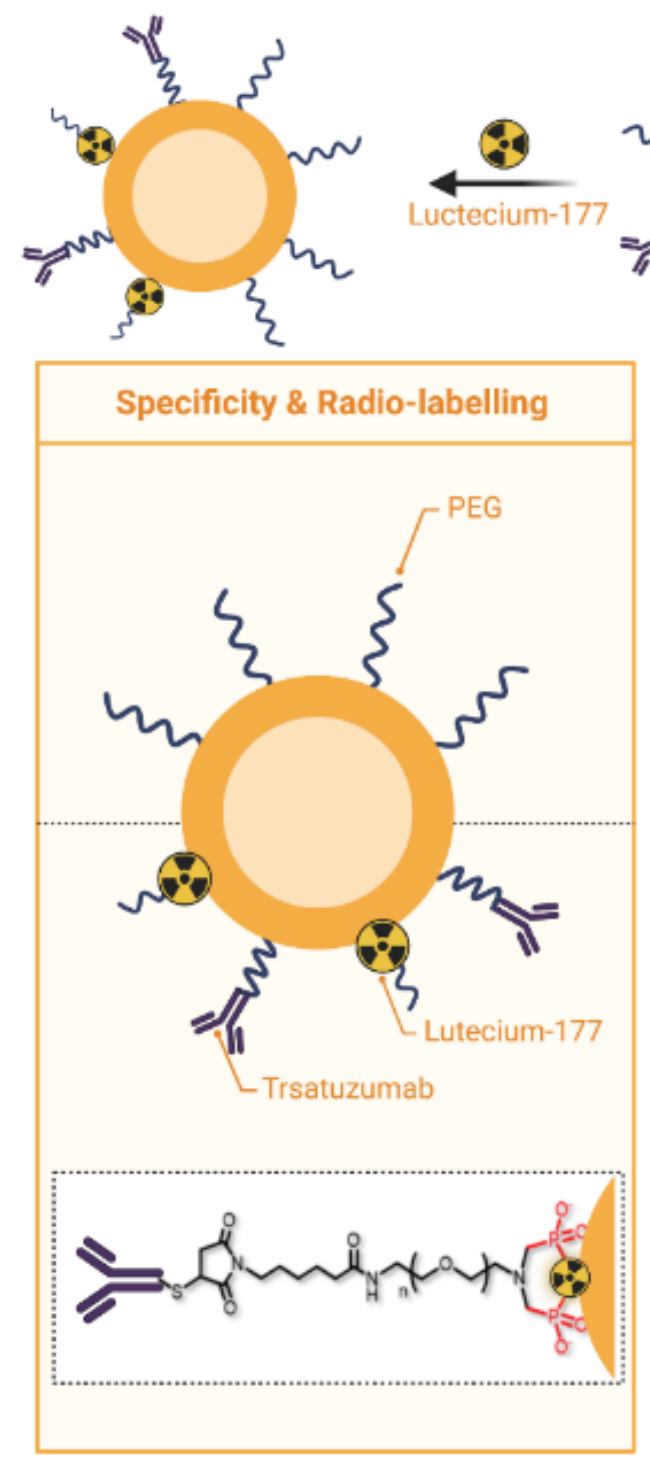

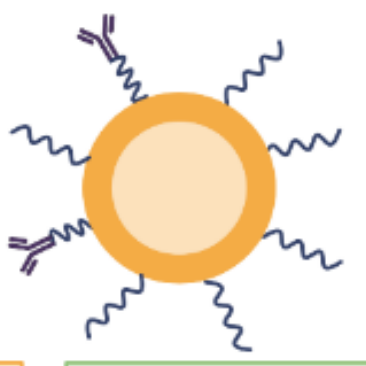

Targeting Metastaic Lymphoma

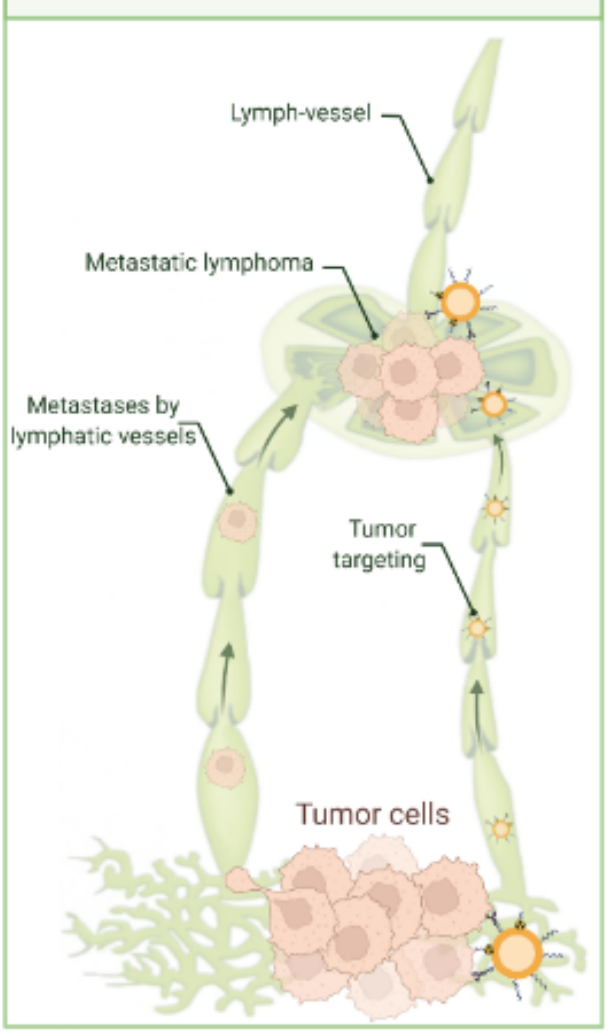

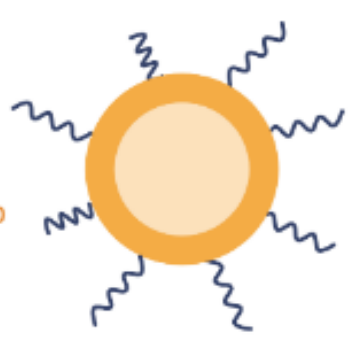
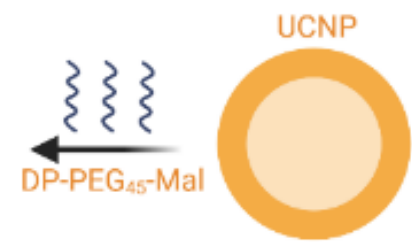

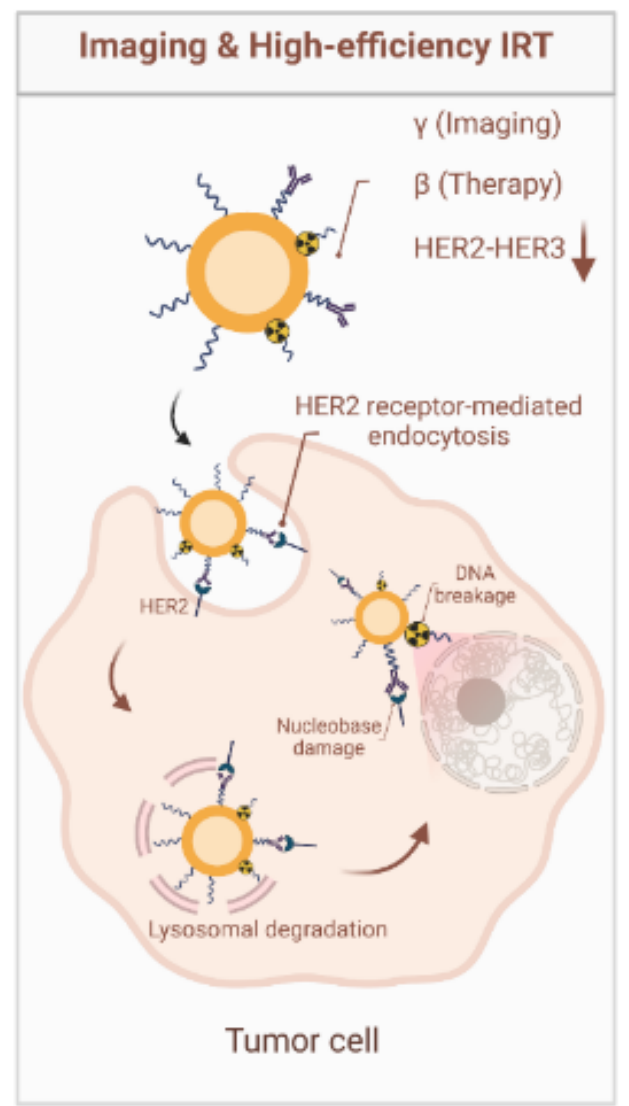

\section{Figure 1}

The schematic diagram of radionuclides ${ }^{177}$ Lu labeled trastuzumab antibody conjugated UCNP (NaGdF4:Yb,Tm@NaLuF4) nanoparticles for lymph nodes metastatic tumor treatment. 
a
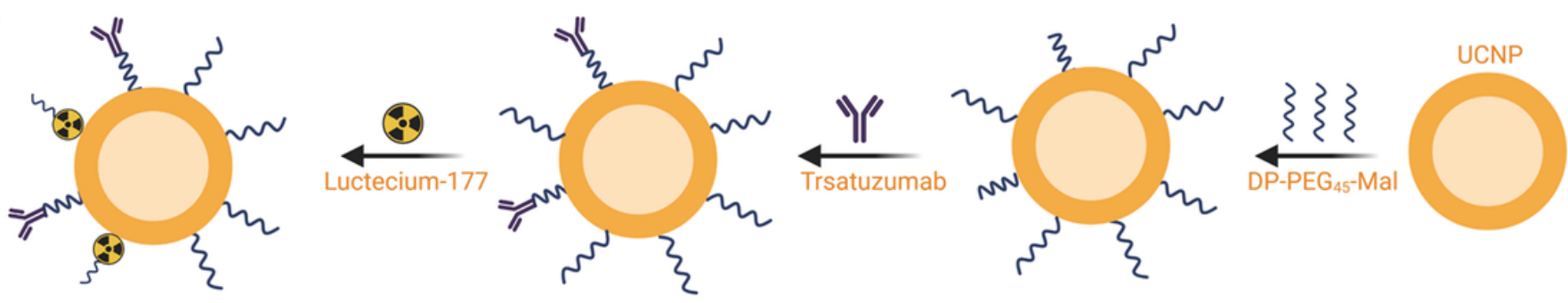

b
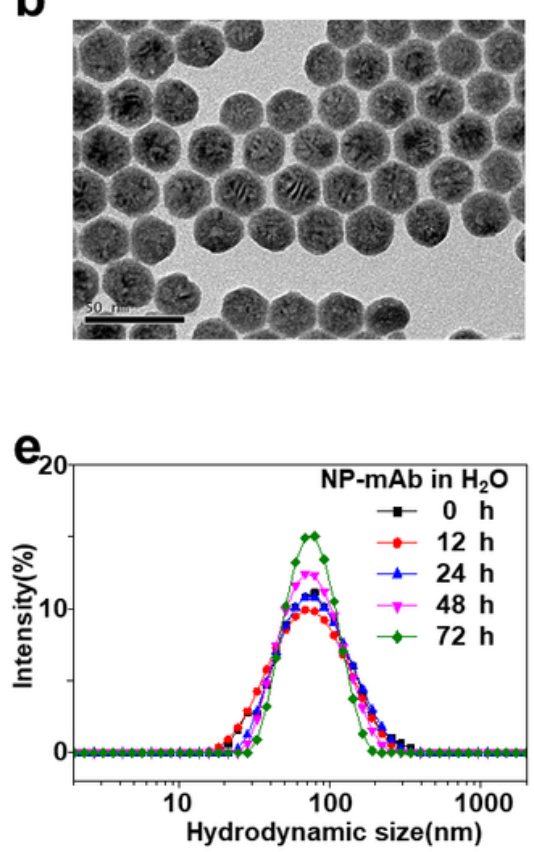
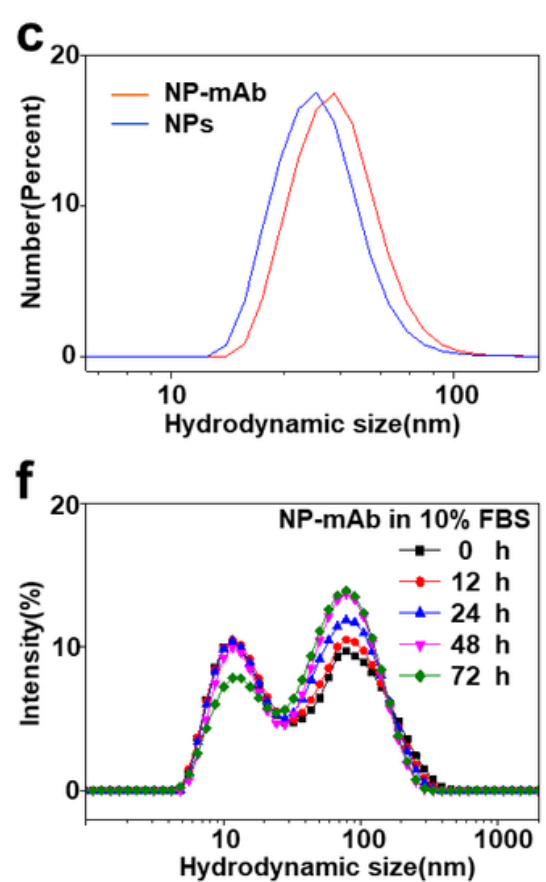

d

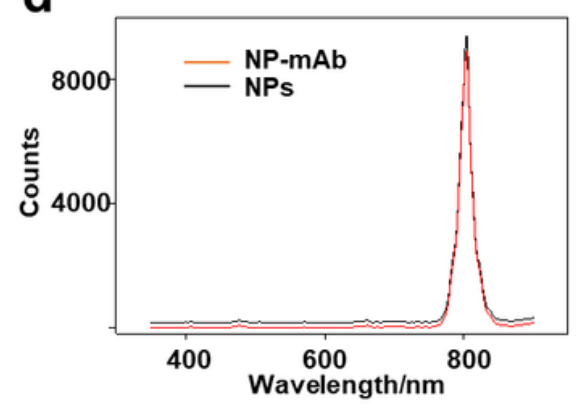

g

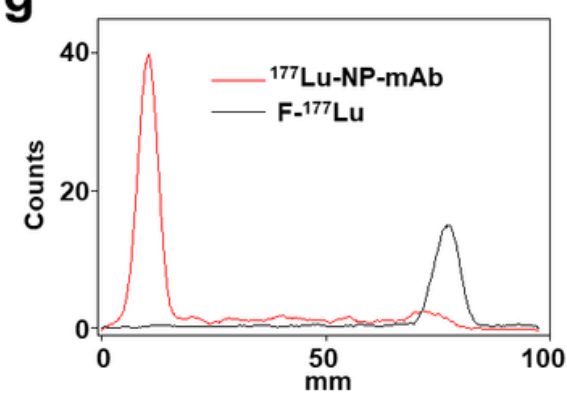

Figure 2

Synthesis and characterization of ${ }^{177}$ Lu-NP-mAb. (a) Schematic depicting the preparation of ${ }^{177}$ Lu-NPmAb. (b)Transmission electron microscopy (TEM) image of $\mathrm{NaGdF}_{4}: \mathrm{Yb}, \mathrm{Tm}_{\mathrm{N}} \mathrm{NaLuF} \mathrm{F}_{4}$ nanoparticles. (c) Hydrodynamic size of NP-mAb and NPs in $\mathrm{H}_{2} \mathrm{O}$ determined by DLS. (d) UV-vis spectra of NP-mAb and NPs under $980 \mathrm{~nm}$ light irradiation. (e-f) Hydrodynamic size of NP-mAb in $\mathrm{H}_{2} \mathrm{O}(\mathrm{e})$ and $10 \% \mathrm{FBS}(\mathrm{f})$ at different time points determined by DLS. (g) Radiochemical pure analysis of ${ }^{177}$ Lu-NP-mAb and F-177 Lu. 

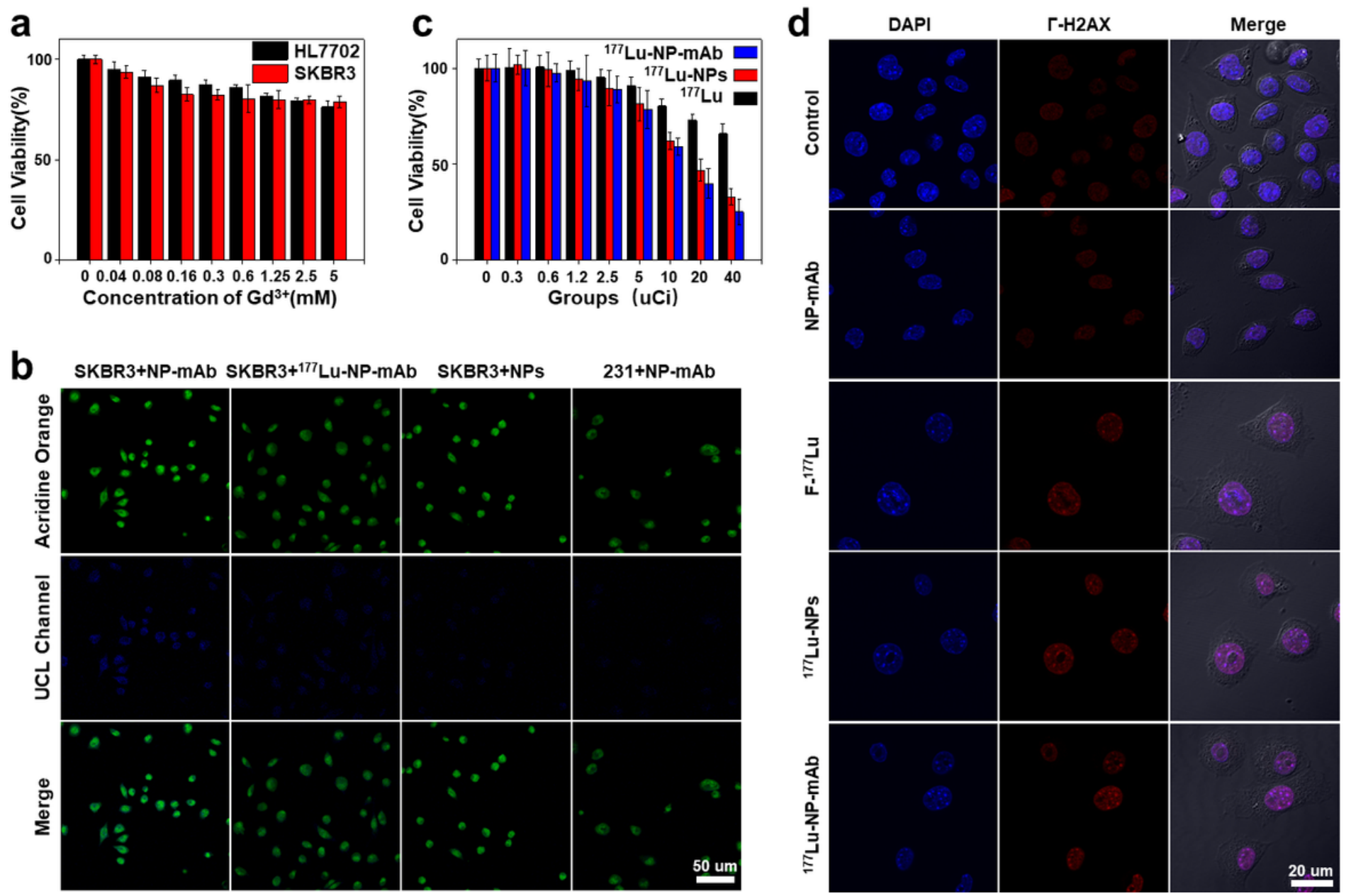

Figure 3

Cell experiments. (a) The cytotoxicity of NP-mAb at different concentrations. (b) Confocal imaging of the HER2 over-expressed cell line (SKBR3) and HER2 low-expressed cell line (MDA-MB-231) incubated with the ${ }^{177}$ Lu-NP-mAb, NP-mAb, respectively (green: acridine orange, blue: UCL Channel, the embedded scale bars correspond to $50 \mu \mathrm{m}$ ). (c) Relative viabilities of SKBR3 cells treated with different doses of free ${ }^{177} \mathrm{Lu},{ }^{177} \mathrm{Lu}-\mathrm{NPs}$ and ${ }^{177} \mathrm{Lu}-\mathrm{NP}-\mathrm{mAb}$ for $24 \mathrm{~h}$. (d) $\mathrm{Y}$-H2AX fluorescence images (blue: DAPI, red: $\mathrm{y}-\mathrm{H} 2 \mathrm{AX}$ ) of SKBR3 cells with different treatments. 

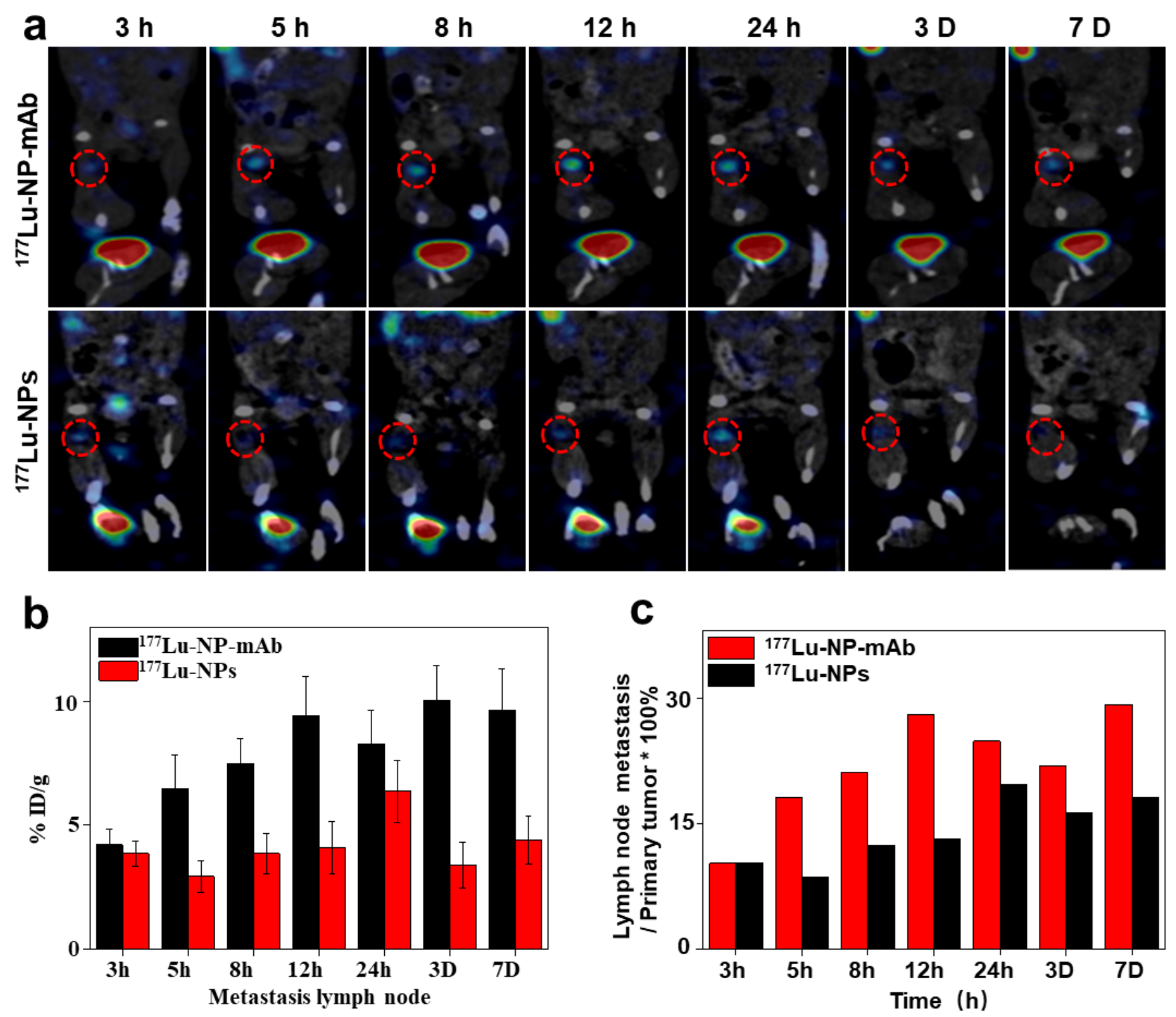

Figure 4

In vivo experiments. (a) SPECT/CT imaging of SKBR3 tumor bearing mice with ${ }^{177}$ Lu-NPs and ${ }^{177}$ Lu-NPmAb injected in the left footpad at different time points. (b) Biodistribution of ${ }^{177}$ Lu-NPs and ${ }^{177}$ Lu-NPmAb in metastasis lymph node were analysed by professional PMOD software (MILabs) at different time points. Error bars represent mean \pm s.d. $(n=3)$. (c) The ratio of ${ }^{177}$ Lu-NPs and ${ }^{177}$ Lu-NP-mAb in metastasis lymph node and primary tumor, analysed by professional PMOD software (MILabs) at different time points. 

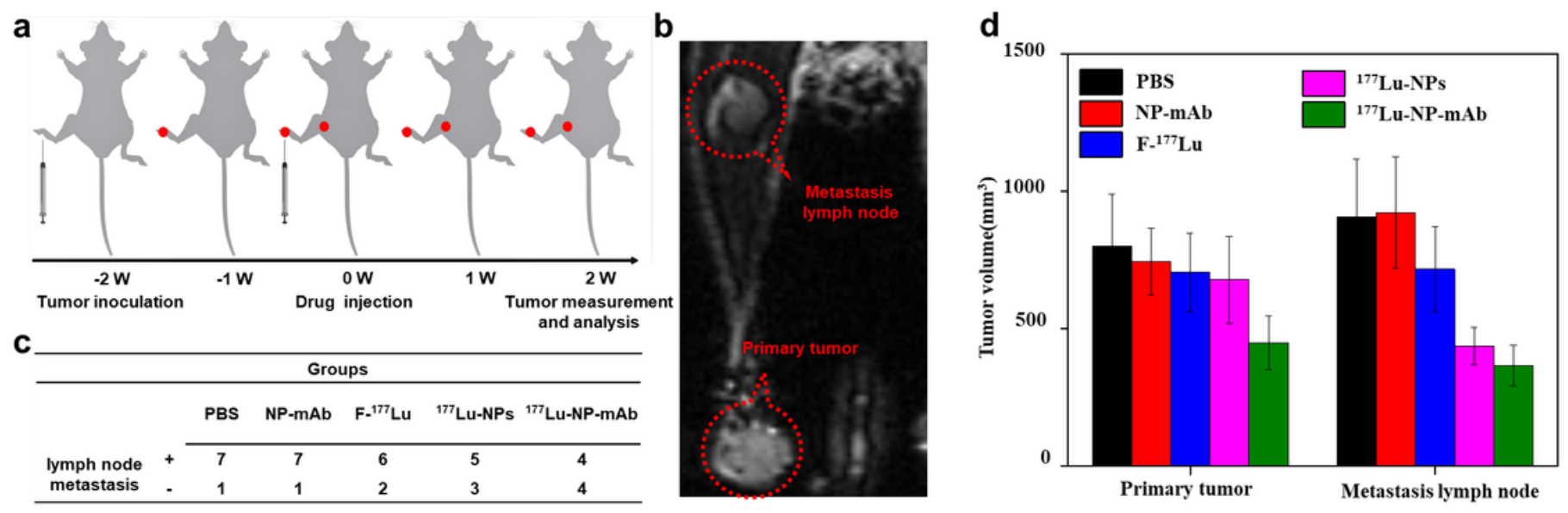

Figure 5

In vivo therapeutic efficacy by radiotherapy. (a) Schematic diagram of radioisotope therapy to suppress primary tumor and metastasis lymph nodes growth. (b) MRI imaging of lymphatic metastasis model mouse. (c) Lymph node metastasis rate of different groups of mice (PBS, NP-mAb, free ${ }^{177}$ Lu, ${ }^{177}$ Lu-NPs, and ${ }^{177}$ Lu-NP-mAb) after 2 weeks of radioisotope therapy. (d) The volume of primary tumor and metastatic lymph nodes in different groups of mice after 2 weeks of radioisotope therapy. 

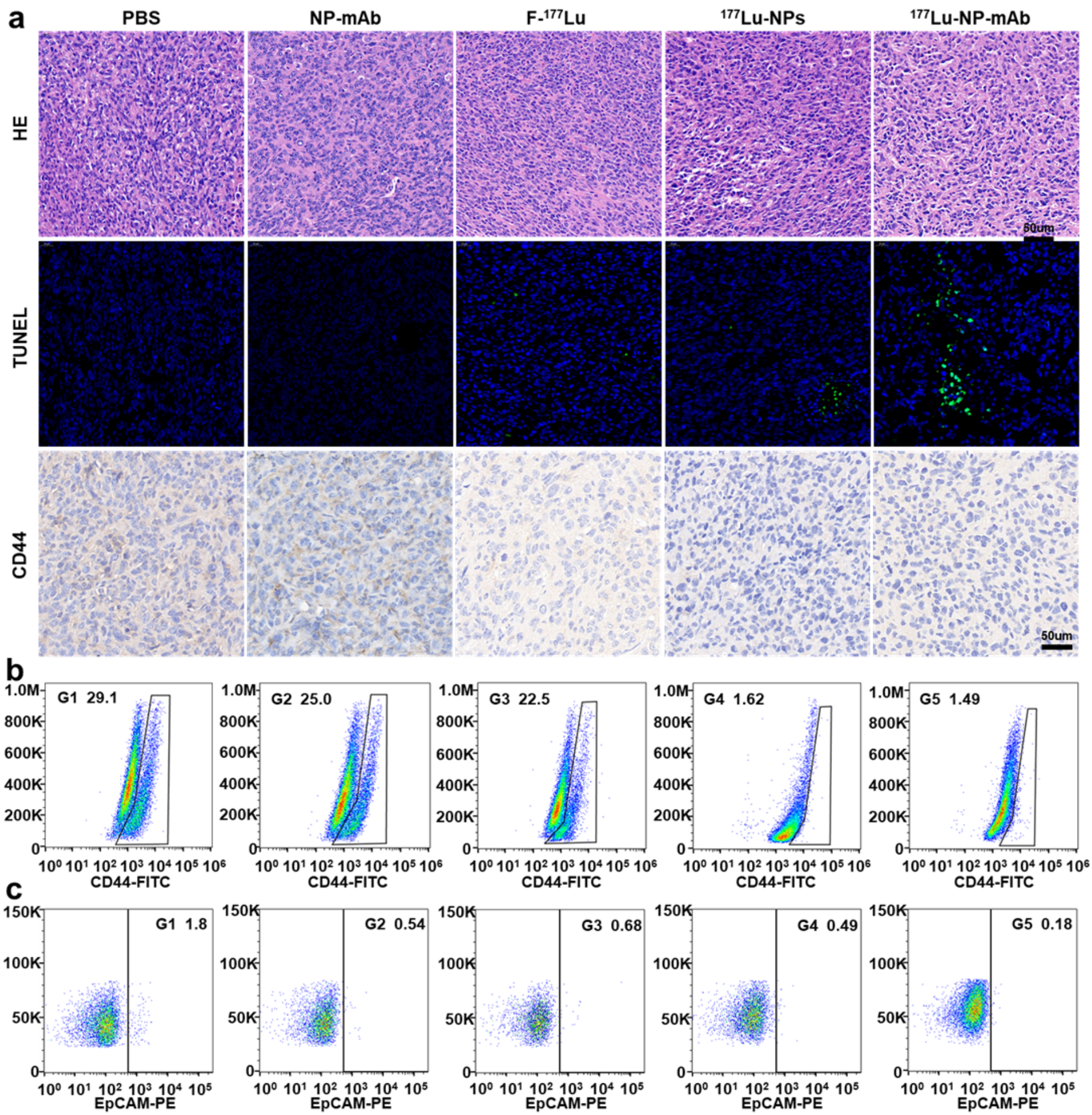

\section{Figure 6}

Histological evaluation and safety assay. (a) Micrographs of H\&E, TUNEL and CD44 stained tumor slices from metastasis lymph nodes of mice with different treatment after 2 weeks. Scale bar:200 $\mu \mathrm{m}$. (b-c) Representative flow cytometry plots showing the CD44 (b) in the metastasis lymph nodes and the EpCAM (c) in the blood from different groups 2 weeks post treatment (G1: PBS, G2: NP-mAb, G3: free ${ }^{177}$ Lu, G4: ${ }^{177}$ Lu-NPs, G5: ${ }^{177}$ Lu-NP-mAb). 


\section{Supplementary Files}

This is a list of supplementary files associated with this preprint. Click to download.

- Graphicalabstract.docx

- SupportingInformation20220115.docx 